(2) OPEN ACCESS

\title{
Anxiety and depression symptoms after COVID-19 infection: results from the COVID Symptom Study app
}

\author{
Kerstin Klaser (D) ,' Ellen J Thompson, ${ }^{2}$ Long H Nguyen, ${ }^{3,4}$ Carole H Sudre, 1,5,6 \\ Michela Antonelli, ${ }^{1}$ Benjamin Murray, ${ }^{1}$ Liane S Canas, ${ }^{1}$ Erika Molteni, ${ }^{1}$ \\ Mark S Graham, ${ }^{1}$ Eric Kerfoot, ${ }^{1}$ Liyuan Chen, ${ }^{1}$ Jie Deng, ${ }_{1}^{1}$ Anna May, ${ }^{7}$ Christina Hu, ${ }^{7}$ \\ Andy Guest, 7 Somesh Selvachandran, ${ }^{7}$ David A Drew, ${ }^{3,4}$ Marc Modat, ${ }^{1}$ \\ Andrew T Chan, ${ }^{3,4}$ Jonathan Wolf, ${ }^{7}$ Tim D Spector, $^{2}$ Alexander Hammers (D) , 1,8 \\ Emma L Duncan, ${ }^{2}$ Sebastien Ourselin, ${ }^{1}$ Claire J Steves (D) ${ }^{2}$
}

\begin{abstract}
- Additional supplemental material is published online only. To view, please visit the journal online (http://dx. doi.org/10.1136/jnnp-2021 327565).
\end{abstract}

For numbered affiliations see end of article.

Correspondence to Dr Claire J Steves, Department of Twin Research and Genetic Epidemiology, King's College London, London SE1 7EH, UK; claire.j.steves@kcl.ac.uk

Received 8 July 2021 Accepted 7 September 2021 Published Online First 28 September 2021

\section{Check for updates}

(c) Author(s) (or their employer(s)) 2021. Re-use permitted under CC BY. Published by BMJ.

To cite: Klaser K, Thompson EJ, Nguyen LH, et al. J Neurol Neurosurg Psychiatry 2021:92:1254-1258.

\section{ABSTRACT}

Background Mental health issues have been reported after SARS-CoV-2 infection. However, comparison to prevalence in uninfected individuals and contribution from common risk factors (eg, obesity and comorbidities) have not been examined. We identified how COVID-19 relates to mental health in the large community-based COVID Symptom Study.

Methods We assessed anxiety and depression symptoms using two validated questionnaires in 413148 individuals between February and April 2021; 26998 had tested positive for SARS-CoV-2. We adjusted for physical and mental prepandemic comorbidities, body mass index (BMI), age and sex.

Findings Overall, $26.4 \%$ of participants met screening criteria for general anxiety and depression. Anxiety and depression were slightly more prevalent in previously SARS-CoV-2-positive (30.4\%) vs SARS-CoV-2-negative (26.1\%) individuals. This association was small compared with the effect of an unhealthy BMI and the presence of other comorbidities, and not evident in younger participants ( $\leq 40$ years). Findings were robust to multiple sensitivity analyses. Association between SARSCoV-2 infection and anxiety and depression was stronger in individuals with recent ( $<30$ days) versus more distant (>120 days) infection, suggesting a short-term effect. Interpretation A small association was identified between SARS-CoV-2 infection and anxiety and depression symptoms. The proportion meeting criteria for self-reported anxiety and depression disorders is only slightly higher than prepandemic.

\section{INTRODUCTION}

Studies from previous coronaviruses suggesting an increased risk of neurological disorders, ${ }^{1}$ and case studies $^{2-4}$ and findings ${ }^{5-7}$ regarding the impact of SARS-CoV-2 infection on the central nervous system $^{8-11}$ led to the hypothesis that anxiety/ depression symptoms may be more prevalent in individuals after SARS-CoV-2 infection. Indeed, several reports suggest that COVID-19 survivors are at increased risk of mood and anxiety disorders 3 months postinfection. ${ }^{12-15}$ Moreover, the Office for National Statistics reported a steep increase in anxiety/depression symptoms in the general public (irrespective of infection status) compared with prepandemic data, adjusting for socioeconomic factors. ${ }^{16}$

Quantifying the relationship of SARS-CoV-2 infection on anxiety/depression symptoms per se requires disentangling the consequences of infection from other factors such as lockdown measures. Direct links to health records may enable assessment of SARS-CoV-2 infection on psychiatric diagnoses $^{15}$; however, it takes time and resources to acquire large cohorts for such longitudinal studies. Alternatively, analysis of self-reported real-time data allows for faster and timely insights into effects on mental health from SARS-CoV-2 infections.

This study aimed to assess prevalence of anxiety/ depression symptoms in individuals with and without prior SARS-CoV-2 infection using a large community cohort, including assessment of other known mental health predictors. We used data from 413148 tested non-healthcare workers who answered a mental health survey between February and April 2021 via the COVID Symptom Study app. ${ }^{17}$

\section{METHODS}

Sample

Data were acquired by the COVID Symptom Study app, ${ }^{17}$ a mobile application developed by health data company Zoe Limited in collaboration with King's College London (KCL), the Massachusetts General Hospital, Lund University and Uppsala University. The app was launched on 24 March 2020 and allows users to report their health status (whether symptomatic or asymptomatic), SARSCoV-2-related testing and results, and vaccination details, daily. On registration, app users provide demographic and clinical data including age, height, weight, sex, comorbidities (ie, cancer, diabetes, eczema, heart disease, lung disease, kidney disease and hay fever) and healthcare worker status. The app contents can also be modified to address arising research questions. We used data from 413148 nonhealthcare worker users who answered a mental health survey between February and April 2021 and reported a SARS-CoV-2 test result. Healthcare workers were excluded from this analysis due to their likely differing pandemic experience. 


\section{Measures}

Between 23 February 2021 and 12 April 2021, app contributors were invited to answer a survey about their mental health. Anxiety/depression symptoms were measured using the Generalised Anxiety Disorder assessment-2 (GAD-2) ${ }^{18}$ and the Patient Health Questionnaire-2 (PHQ-2). ${ }^{19}$ These measures examine symptoms in the preceding 2 weeks, each using two questions. For each question regarding frequency of a proposed situation/ feeling, users can answer 'not at all', 'several days', 'more than half the days' or 'nearly every day'. Each answer scores from 0 for 'not at all' to 3 for 'nearly every day'. Each questionnaire has a score ranging from 0 to 6 . Previous studies have shown an optimal cut-off point for possible anxiety or depression disorder of $\geq 3$, yielding a sensitivity of $>80 \% .{ }^{18}{ }^{19}$ A binary outcome variable was created by grouping those who scored $\geq 3$ in either GAD-2 or PHQ-2, or $<3$.

Logistic regression was used to study whether mental health status was associated with a positive SARS-CoV-2 test result. We adjusted for age, sex, body mass index (BMI) groups (underweight, overweight, obese, with normal BMI (18.5-24.9) as the reference), and comorbidities including learning disabilities; and applied inverse probability weighting for the probability of getting tested for SARS-CoV-2. Data are presented using descriptive statistics. Additionally, logistic regressions were employed stratifying by age groups $(18-30,31-40,41-50,51-60,61-70$ and $>70)$. A sensitivity analysis was performed stratifying by presence of prepandemic mental health disorders (see online supplemental materials).

Further analyses in SARS-CoV-2 affected individuals assessed for association with days since infection confirmation and anxiety/depression symptoms, using days between positive test date and date of survey, grouped into:<30, 30-60, 60-90,
90-120 and >120 days. Individuals reporting a positive test result $>120$ days before answering the survey served as reference.

Data were extracted and preprocessed with ExeTera, ${ }^{20}$ a Pandas-like library developed at KCL, and statistical analysis was performed using Python (Pandas, NumPy and SciPy).

\section{RESULTS}

Between 23 February and 12 April 2021, 421977 non-healthcare workers (aged 18-99 years, BMI 15-55) answered the mental health survey and logged a SARS-CoV-2 test result (386150 negative, 35827 positive). A total of 26998 positive tests were PCR or lateral flow results; positive antibody tests (8829) were excluded as time of infection was unknown.

Of the total participants, $26.4 \%$ (109 116) scored $\geq 3$ in GAD-2 and/or PHQ-2. Participants with anxiety/depression symptoms were younger, had more comorbidities and were more often female, compared with unaffected individuals. Among those predicted to have anxiety or depression (based on a score of $\geq 3$ on GAD-2 or PHQ-2), 38.06\% (41 525) reported a previous diagnosis of a prepandemic mental health disorder and $5.79 \%(6320)$ reported a learning disability. The study population's demographic characteristics are presented in table 1.

SARS-CoV-2 infection was associated with anxiety/depression symptoms (OR 1.08, 95\% CI 1.07 to $1.10, \mathrm{p}<0.001$ ). However, stronger associations with anxiety/depression symptoms were observed for unhealthy BMI categories (ie, underweight, overweight and obese) with ORs of 1.26 (95\% CI 1.22 to 1.30 , $\mathrm{p}<0.001), 1.21(95 \%$ CI 1.20 to $1.22, \mathrm{p}<0.001)$ and $1.61(95 \%$ CI 1.59 to $1.62, \mathrm{p}<0.001)$, respectively. Participants reporting one or more comorbidities (OR $1.25,95 \%$ CI 1.24 to 1.26 , $\mathrm{p}<0.001$ ), and those with learning disabilities (OR 1.35, 95\% CI

Table 1 Demographic characteristics

\begin{tabular}{|c|c|c|c|}
\hline & $\begin{array}{l}\text { Anxiety or depression symptoms: yes } \\
\mathrm{n}=109116(26.4 \%)\end{array}$ & $\begin{array}{l}\text { Anxiety or depression symptoms: no } \\
\mathrm{n}=304032(73.6 \%)\end{array}$ & $\begin{array}{l}\text { Total } \\
\mathrm{N}=413148\end{array}$ \\
\hline Age (years), mean (SD) & $50.7(13.9)$ & $55.6(12.9)$ & $54.1(13.4)$ \\
\hline Underweight (BMI <18.5) & $1459(1.34 \%)$ & $3190(1.05 \%)$ & $4649(1.13 \%)$ \\
\hline Normal weight (BMI 18.6-24.9) & $40816(37.41 \%)$ & $134695(44.30 \%)$ & $175511(42.48 \%)$ \\
\hline Overweight (BMI 25-29.9) & $36012(33.00 \%)$ & $106455(35.01 \%)$ & $142467(34.48 \%)$ \\
\hline Obese (BMI >30) & $30829(28.25 \%)$ & $59692(19.63 \%)$ & $90521(21.91 \%)$ \\
\hline Previously diagnosed with mental health condition & $41525(38.06 \%)$ & $57067(18.77 \%)$ & $98592(23.86 \%)$ \\
\hline No previous mental health condition & $67591(61.94 \%)$ & $246965(81.23 \%)$ & $314556(76.14 \%)$ \\
\hline Learning disability: yes & $6320(5.79 \%)$ & $9987(3.28 \%)$ & $16307(3.95 \%)$ \\
\hline Learning disability: no & $102796(94.21 \%)$ & $294045(96.72 \%)$ & $396841(96.05 \%)$ \\
\hline Female & $80446(73.73 \%)$ & $200563(65.97 \%)$ & $281009(68.02 \%)$ \\
\hline $\begin{array}{l}\text { Positive COVID-19 } \\
30-60 \text { days before survey }\end{array}$ & $2149(26.15 \%)$ & $5043(26.85 \%)$ & $7192(26.64 \%)$ \\
\hline $\begin{array}{l}\text { Positive COVID-19 } \\
60-90 \text { days before survey }\end{array}$ & $1843(22.42 \%)$ & $4505(23.99 \%)$ & $6348(23.51 \%)$ \\
\hline $\begin{array}{l}\text { Positive COVID-19 } \\
\text { 90-120 days before survey }\end{array}$ & $1162(14.14 \%)$ & $2791(14.86 \%)$ & $3953(14.64 \%)$ \\
\hline $\begin{array}{l}\text { Positive COVID-19 } \\
<120 \text { days before survey }\end{array}$ & $1751(21.30 \%)$ & $3960(21.09 \%)$ & $5711(21.15 \%)$ \\
\hline
\end{tabular}

BMI, body mass index. 

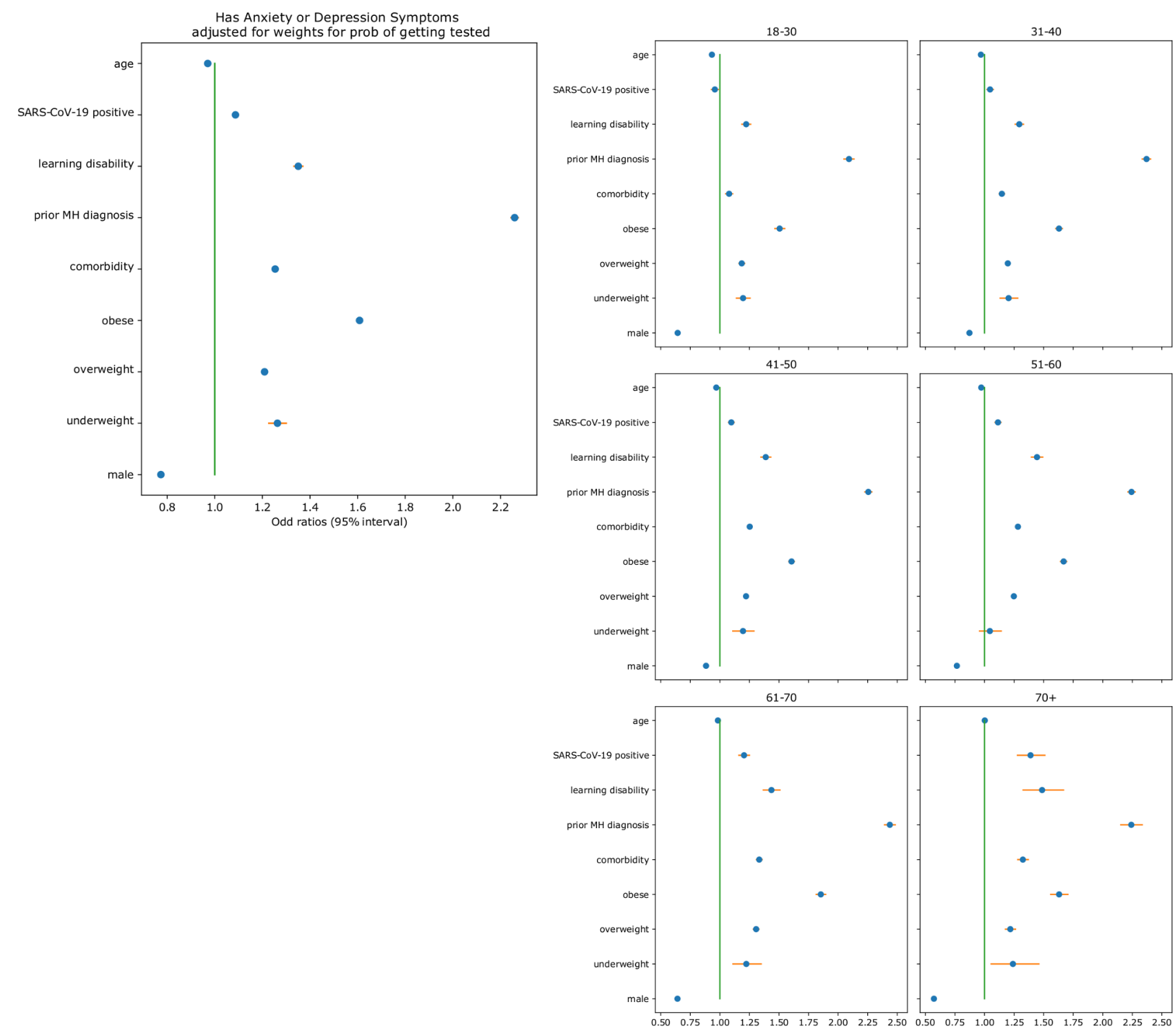

Figure 1 Association between age, body mass index, male sex, comorbidities, a previous diagnosis of a MH condition, learning disabilities, a positive SARS-CoV-2 test result (PCR and lateral flow) and the OR of anxiety/depression symptoms suggested by the results of the $\mathrm{MH}$ survey. $\mathrm{MH}$, mental health.

1.33 to $1.37, \mathrm{p}<0.001)$ were more likely to have anxiety/ depression symptoms. Individuals reporting a previously diagnosed mental health condition had the highest odds of reporting anxiety/depression symptoms (OR 2.26, 95\% CI 2.24 to 2.28 , $\mathrm{p}<0.001$ ) (figure 1).

We observed no significant difference in the small overall increased odds of anxiety/depression symptoms with SARSCoV-2 infection in those with a history of prior mental health conditions (OR 1.09, 95\% CI 1.06 to $1.12, \mathrm{p}<0.001)$ and those without such prior history of (OR $1.09,95 \%$ CI 1.07 to 1.10 , $\mathrm{p}<0.001)$.

Stratification by age group showed no association between a positive SARS-CoV-2 test and anxiety/depression symptoms in young groups ( $<40$ years). Other variables (sex, comorbidities and BMI) were consistent in age-stratified analyses. Finally, in the 26998 cases positive for SARS-CoV-2 by PCR and lateral flow, we tested whether elapsed time after a positive test affected mental health. The relationship between SARS-CoV-2 and anxiety/depression symptoms changed over time, with increased risk of anxiety/depression symptoms in those diagnosed $<30$ days compared with those diagnosed $>120$ days prior to the survey (OR $1.15,95 \%$ CI 1.10 to $1.2, \mathrm{p}<0.001$ ).

\section{DISCUSSION}

In this large, community-based study, we report a small positive association between SARS-CoV-2 infection and anxiety/depression symptoms. However, this was dwarfed by associations with the known risk factors BMI, sex and comorbidities. Results were robust to sensitivity analyses stratifying by prior mental health disorder diagnoses. Further, no association between SARS$\mathrm{CoV}-2$ infection and anxiety/depression symptoms was found in younger age groups ( $<40$ years).

Association between SARS-CoV-2 infection and anxiety/ depression symptoms changed over time, with the strongest association in those infected $<30$ days prior to the survey, suggesting a short-term effect of infection on mental health only. It is possible that other factors affecting mental health which were changing over the pandemic (eg, lockdown) may moderate an effect of elapsed time since SARS-CoV-2 infection on mental health. ${ }^{21}$ 
Overall prevalence of anxiety/depression symptoms in our study $(26.4 \%)$ is slightly increased compared with prepandemic levels of mental health issues in the UK general population assessed by the UK Household Longitudinal Study with the GHQ-12 questionnaire (18.9\% in $2018^{22}$ ) but broadly comparable to the level seen in April $2021\left(27.3 \%{ }^{22}\right)$. This previous 2021 study did not explore any relationship with SARS-CoV-2 infection. A recent analysis of 1112 subjects experiencing probable COVID-19 symptoms suggested a positive association between COVID-19 and anxiety/depression symptoms 1-7 months after suggested infection (OR 1.31-1.47). ${ }^{23}$ Our study benefits from a much larger sample size of tested participants.

Our study has several limitations. Data are self-reported using a mobile app and may disproportionately represent more affluent populations. We only had one time point of mental health data collection, limiting our ability to test if associations changed as the pandemic progressed. Additionally, although we applied weighting for the probability of being tested for the virus, results referring to time since testing might be still biased due to limited testing capacity early in the pandemic. As in any study assessing mental health through questionnaires, selection bias (whereby mental health influences who responds) and reporting bias (relating to perception and/or influence of a 'valid' reason to report) may limit the validity of our results. Further analyses of longitudinal datasets with different reporting structures are warranted.

\section{CONCLUSION}

This study suggests a weak association between SARS-CoV-2 infection and anxiety/depression symptoms, especially in adults aged $>40$ years, which is small relative to known risk factors such as previous medical or mental health conditions and/or unhealthy BMI. The association was most evident in recently infected individuals. This suggests that an effect of SARS-CoV-2 on mental health may be only of short duration. Further exploration may help to understand factors that will improve mental health after SARS-CoV-2 infection.

\section{Author affiliations \\ ${ }^{1}$ School of Biomedical Engineering \& Imaging Sciences, King's College London, London, UK \\ 'Department of Twin Research and Genetic Epidemiology, King's College London, London, UK \\ ${ }^{3}$ Clinical \& Translational Epidemiology Unit, Massachusetts General Hospital and Harvard Medical School, Harvard Medical School, Boston, Massachusetts, USA ${ }^{4}$ Division of Gastroenterology, Department of Medicine, Massachusetts General Hospital and Harvard Medical School, Harvard Medical School, Boston, Massachusetts, USA \\ ${ }^{5}$ Department of Medical Physics and Bioengineering, UCL Centre for Medical Image Computing (CMIC), London, UK \\ ${ }^{6} \mathrm{MRC}$ Unit for Lifelong Health and Ageing, Department of Population Science and Experimental Medicine, University College London, London, UK \\ ${ }^{7}$ Zoe Limited, London, UK \\ ${ }^{8}$ PET Centre, Division of Imaging Sciences and Biomedical Engineering, King's College London, London, UK}

\section{Twitter Ellen J Thompson @EllenJoT}

Contributors KK performed the analyses; KK, EJT, LHN, AM, CH and CJS worked at conceptualisation and methodology: KK, BM, EK, LC, JD and MM performed the data extraction and curation; KK, EJT, ELD, AH and CJS wrote the manuscript; CH, SS, AM and AG developed the data collection system; TDS, SO and CJS conceived the CSS and obtained funds; SO and CJS coordinated this research. All the authors critically reviewed the manuscript and had access to the COVID Symptom Study dataset, which is also accessible to researchers in the public interest.

Funding This work is supported by the Wellcome Engineering and Physical Sciences Research Council Centre for Medical Engineering at King's College London (WT203148/Z/16/Z) and the UK Department of Health via the National Institute for Health Research (NIHR) comprehensive Biomedical Research Centre award to
Guy's \& St Thomas' NHS Foundation Trust in partnership with King's College London and King's College Hospital NHS Foundation Trust, the Medical Research Council (MRC) and British Heart Foundation. SO and MM are supported by the UK Research and Innovation London Medical Imaging \& Artificial Intelligence Centre for Value Based Healthcare, and the Wellcome Flagship Programme (WT213038/Z/18/Z). This research was funded in part by the Wellcome Trust (WT213038/Z/18/Z and WT203148/Z/16/Z). For the purpose of open access, the author has applied a CC BY public copyright license to any author accepted manuscript version arising from this submission. EM is funded by an MRC Skills Development Fellowship Scheme at KCL. CHS, EJT and CJS are supported by the National Core Studies, an initiative funded by UKRI, NIHR and the Health and Safety Executive, and funded by the Medical Research Council (MC_PC_20030). CHS is also supported by an Alzheimer's Society Junior Fellowship (AS-JF-17-011). Zoe Limited supported all aspects of building and running the app and service to all users worldwide. ATC is a Stuart and Suzanne Steele MGH Research Scholar. LHN is supported by an NIH K23DK125838 award, the American Gastroenterological Association Research Scholars Award, and the Crohn's and Colitis Foundation Career Development Award. DAD and LHN are supported by the AGA-Takeda COVID-19 Rapid Response Research Award (AGA2021-5102). DAD is supported by NIH/NIDDK K01DK120742. ATC, LHN, and DAD are supported by the Massachusetts Consortium on Pathogen Readiness. We express our sincere thanks to all the participant users of the app, including study volunteers enrolled in cohorts within the Coronavirus Pandemic Epidemiology consortium. We thank the staff of Zoe Limited, the Department of Twin Research at King's College London, the Clinical \& Translational Epidemiology Unit at Massachusetts General Hospital, Researchers and staff at Lund University in Sweden for their tireless work in contributing to the running of the study and data collection.

Disclaimer The COVID Symptom Study app was developed by Zoe Limited for data collection as a not-for-profit effort. The funder had no role in study design, data analysis, data interpretation or writing of the manuscript. The corresponding author had the final responsibility for the decision to submit for publication.

Competing interests $A M, C H, A G, S S$ and JW are employees of Zoe Limited. TDS reports being a consultant for Zoe Limited during the conduct of the study. ATC previously served as an investigator on a separate study supported by Zoe Limited.

Patient consent for publication Not applicable.

Ethics approval Ethical approval for use of the app for research purposes in the UK was granted by the KCL Ethics Committee (review reference LRS-19/20-18210); all app users provided consent for non-commercial use.

Provenance and peer review Not commissioned; externally peer reviewed.

Supplemental material This content has been supplied by the author(s). It has not been vetted by BMJ Publishing Group Limited (BMJ) and may not have been peer-reviewed. Any opinions or recommendations discussed are solely those of the author(s) and are not endorsed by BMJ. BMJ disclaims all liability and responsibility arising from any reliance placed on the content. Where the content includes any translated material, BMJ does not warrant the accuracy and reliability of the translations (including but not limited to local regulations, clinical guidelines, terminology, drug names and drug dosages), and is not responsible for any error and/or omissions arising from translation and adaptation or otherwise.

Open access This is an open access article distributed in accordance with the Creative Commons Attribution 4.0 Unported (CC BY 4.0) license, which permits others to copy, redistribute, remix, transform and build upon this work for any purpose, provided the original work is properly cited, a link to the licence is given, and indication of whether changes were made. See: https://creativecommons.org/ licenses/by/4.0/.

\section{ORCID iDs}

Kerstin Klaser http://orcid.org/0000-0002-5230-6939

Alexander Hammers http://orcid.org/0000-0001-9530-4848

Claire J Steves http://orcid.org/0000-0002-4910-0489

\section{REFERENCES}

1 Rogers JP, Chesney E, Oliver D, et al. Psychiatric and neuropsychiatric presentations associated with severe coronavirus infections: a systematic review and meta-analysis with comparison to the COVID-19 pandemic. Lancet Psychiatry 2020;7:611-27.

2 Ellul MA, Benjamin L, Singh B, et al. Neurological associations of COVID-19. Lancet Neurol 2020;19:767-83.

3 Varatharaj A, Thomas N, Ellul MA, et al. Neurological and neuropsychiatric complications of COVID-19 in 153 patients: a UK-wide surveillance study. Lancet Psychiatry 2020;7:875-82.

4 Paterson RW, Brown RL, Benjamin L, et al. The emerging spectrum of COVID-19 neurology: clinical, radiological and laboratory findings. Brain 2020;143:3104-20.

5 Kremer S, Lersy F, Anheim M, et al. Neurologic and neuroimaging findings in patients with COVID-19: a retrospective multicenter study. Neurology 2020;95:e1868-82.

6 Pezzini A, Padovani A. Lifting the mask on neurological manifestations of COVID-19. Nat Rev Neurol 2020;16:636-44 
7 Raman B, Cassar MP, Tunnicliffe EM, et al. Medium-term effects of SARS-CoV-2 infection on multiple vital organs, exercise capacity, cognition, quality of life and mental health, post-hospital discharge. EClinicalMedicine 2021;31:100683.

8 ladecola C, Anrather J, Kamel H. Effects of COVID-19 on the nervous system. Cell 2020;183:16-27.

9 Kreye J, Reincke SM, Prüss H. Do cross-reactive antibodies cause neuropathology in COVID-19? Nat Rev Immunol 2020;20:645-6.

10 Meinhardt J, Radke J, Dittmayer C, et al. Olfactory transmucosal SARS-CoV-2 invasion as a port of central nervous system entry in individuals with COVID-19. Nat Neurosci 2021;24:168-75.

11 Rhea EM, Logsdon AF, Hansen KM, et al. The S1 protein of SARS-CoV-2 crosses the blood-brain barrier in mice. Nat Neurosci 2021;24:368-78.

12 Holmes EA, O'Connor RC, Perry VH, et al. Multidisciplinary research priorities for the COVID-19 pandemic: a call for action for mental health science. Lancet Psychiatry 2020;7:547-60.

13 Vindegaard N, Benros ME. COVID-19 pandemic and mental health consequences: systematic review of the current evidence. Brain Behav Immun 2020:89:531-42.

14 Taquet M, Geddes JR, Husain M, et al. 6-month neurological and psychiatric outcomes in 236379 survivors of COVID-19: a retrospective cohort study using electronic health records. The Lancet Psychiatry 2021;8:416-27.

15 Taquet M, Luciano S, Geddes JR, et al. Bidirectional associations between COVID-19 and psychiatric disorder: retrospective cohort studies of 62354 COVID-19 cases in the USA. Lancet Psychiatry 2021;8:130-40.
16 Office for National Statistics. Coronavirus and depression in adults, Great Britain: January to March 2021. Available: https://www.ons.gov.uk/peoplepo pulationandcommunity/wellbeing/articles/coronavirusanddepressioninadultsgrea tbritain/januarytomarch2021

17 Menni C, Valdes AM, Freidin MB, et al. Real-time tracking of self-reported symptoms to predict potential COVID-19. Nat Med 2020;26:1037-40.

18 Kroenke K, Spitzer RL, Williams JBW, et al. Anxiety disorders in primary care: prevalence, impairment, comorbidity, and detection. Ann Intern Med 2007:146:317-25.

19 Kroenke K, Spitzer RL, Williams JBW. The patient health Questionnaire-2: validity of a two-item depression screener. Med Care 2003:41:1284-92.

20 Murray B, Kerfoot E, Graham MS, et al. Accessible data curation and analytics for international-scale citizen science datasets. arXiv 2020

21 O'Connor RC, Wetherall K, Cleare S, et al. Mental health and well-being during the COVID-19 pandemic: longitudinal analyses of adults in the UK COVID-19 Mental Health \& Wellbeing study. Br J Psychiatry 2021;218:326-33.

22 Pierce M, Hope H, Ford T, et al. Mental health before and during the COVID-19 pandemic: a longitudinal probability sample survey of the UK population. Lancet Psychiatry 2020;7:883-92

23 Niedzwiedz CL, Benzeval M, Hainey K, et al. Psychological distress among people with probable COVID-19 infection: analysis of the UK household longitudinal study. BJPsych Open 2021;7:E104. 